\title{
Utilização de dispositivo móvel com Realidade Aumentada: um estudo de caso na Educação Infantil com o aplicativo Cubo Kids
}

\author{
Ceres G. B. Morais ${ }^{1}$, Carlos Ramon S. da Silva ${ }^{1}$, Antônio Kalielso S. de Mendonça ${ }^{2}$ \\ ${ }^{1}$ Departamento de Informática - Universidade do Estado do Rio Grande do Norte (UERN) \\ CEP: 59.600-000 - Mossoró - RN - Brasil \\ ${ }^{2}$ Núcleo de Educação a Distância - Universidade Federal Rural do Semi-Árido (UFERSA) \\ CEP: 59.600-000 - Mossoró - RN - Brasil \\ \{ceresgbmorais, c.ramon.s.s, kalielson\}@gmail.com
}

\begin{abstract}
Mobile devices have had important role by changing how people have access to information. Their use in educational purposes has been growing every day and it is noticed how Augmented Reality may contribute in application development in that area. This work presents a case study that took place in child education groups, in a school of Mossoró city that used the application Cubo Kids, which was developed with Augmented Reality technology in order to enlarge teaching and learning possibilities. The research showed that the application may bring great possibilities to children teaching in all child educational levels.
\end{abstract}

Resumo. Os dispositivos móveis vêm tendo um papel importante nas mudanças de como as pessoas têm acesso às informações. A utilização de dispositivos móveis com finalidades educativas vem crescendo a cada dia e, aliada a ela, nota-se o quanto a Realidade Aumentada pode contribuir no desenvolvimento de aplicativos desta área. Este trabalho apresenta um estudo de caso realizado em turmas de Educação Infantil, de uma escola da cidade de Mossoró/RN, utilizando o aplicativo Cubo Kids, o qual foi desenvolvido com a tecnologia de Realidade Aumentada, com a finalidade de permitir ampliar as possibilidades de ensino e aprendizagem. Com a pesquisa, verificou-se que o aplicativo pode trazer grandes possibilidades ao ensino de crianças de todos os níveis da Educação Infantil.

\section{Introdução}

Cada vez mais presente no cotidiano, os dispositivos móveis vêm tendo um papel importante nas mudanças de como as pessoas têm acesso às informações, como se relacionam, como aprendem. Utilizar smartphones e tablets vem se tornado tão comum na sala de aula, quanto utilizar lápis, livros e caderno, principalmente no tocante à realização de pesquisas ou como material didático, haja vista a rapidez e forma prática de como as informações são conseguidas por meio dessas ferramentas.

Nesse contexto, de utilização de dispositivos móveis para o aprendizado, surge o termo Aprendizagem Móvel (Mobile Learning ou M-Learning), que tem por finalidade possibilitar o uso de dispositivos móveis e suas ferramentas para o desenvolvimento de atividades de ensino e aprendizagem, não apenas no espaço escolar, mas em todos os espaços sociais. Assim, a mobilidade não é apenas física, mas social [Lima et al, 2016]

Aliada ao conceito de M-Learning, existem várias opções tecnológicas que

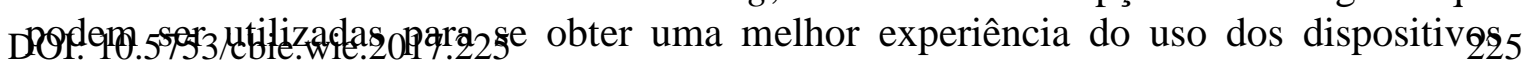
móveis no ensino. Dentre elas pode-se destacar que a Realidade Aumentada (RA) tem 
sido bastante utilizada para o desenvolvimento do conhecimento, tornando prática e dinâmica a interatividade do usuário juntamente com a participação da mobilidade oferecida pelos dispositivos móveis no desenvolvimento do conhecimento [Tori, 2006].

Tendo em vista o que foi exposto acima, este trabalho apresenta como pode-se fazer a utilização de dispositivos móveis com Realidade Aumentada no contexto educacional, através de um estudo de caso realizado em turmas da Educação Infantil com o uso do aplicativo Cubo Kids.

O artigo está organizado da seguinte maneira: a Seção 2 apresenta os conceitos relacionados ao estudo; a Seção 3 apresenta a ferramenta Cubo Kids; a Seção 4 apresenta o estudo de caso desenvolvido; a Seção 5 aborda os resultados coletados durante o estudo de caso; e, por fim, na Seção 6, são apresentadas as considerações finais do trabalho.

\section{Aprendizagem Móvel e Realidade Aumentada}

Aprendizagem Móvel é um paradigma que consiste no ensino e aprendizado através de dispositivos móveis, como smartphones e tablets, dentre outros, de maneira tam que possa priorizar a mobilidade dos indivíduos e proporcionar um aprendizado mais dinâmico e flexível [Attewell et. al 2005]. O termo aprendizagem móvel surge em uma perspectiva recente, com a chegada das tecnologias portáteis, que possibilitam a comunicação e interação por meio de recursos computacionais [Saccol; Schlemmer; Barbosa, 2011]. Com o uso cada vez maior de dispositivos móveis de acesso à Internet, como tablets, smartphones e laptops, ampliam-se também as formas de uso desses recursos para fins educativos, dentro e fora do ambiente escolar [Lima et al, 2016].

De acordo com Kukulska-Hulme (2005), a M-Learning pode ser definida como qualquer disposição de ensino onde as tecnologias dominantes são as ferramentas móveis. Woodill (2010) sugere que ela é vista como uma maneira de manter as pessoas em contato entre si e com as fontes de informação, independentemente de onde quer que estejam localizadas. Para agregar valores e conhecimento, a educação tem se apoiado às inovações tecnológicas e à disposição de recursos que visam explorar o conhecimento de forma curiosa e intuitiva a partir de jogos e aplicativos que utilizam da Realidade Aumentada [Oliveira et al, 2016].

Realidade Aumentada (RA) é a sobreposição de objetos virtuais gerados por computador num ambiente real, utilizando para isso algum dispositivo tecnológico [Kirner e Pinho 2006]. Apesar de parecer uma tecnologia nova a base para a realidade aumentada surgiu na década de 1960, com o pesquisador Ivan Sutherland, onde escreveu um artigo sobre a evolução da realidade aumentada e seus reflexos no mundo real [Sutherland 1964] e desenvolveu um capacete de visão óptica direta rastreando para visualização de objetos 3D no ambiente real.

O primeiro projeto de realidade aumentada foi desenvolvido na década de 1980 pela Força Aérea Americana, com visão óptica direta, misturando elementos virtuais com o ambiente físico do usuário, constituindo um cockpit de avião [Kirner 2008]. Para melhor entendimento, a Figura 1 representa o funcionamento de uma aplicação com uso da Realidade Aumentada descrita por Cardoso et al (2014). 
VI Congresso Brasileiro de Informática na Educação (CBIE 2017)

Anais do XXIII Workshop de Informática na Escola (WIE 2017)

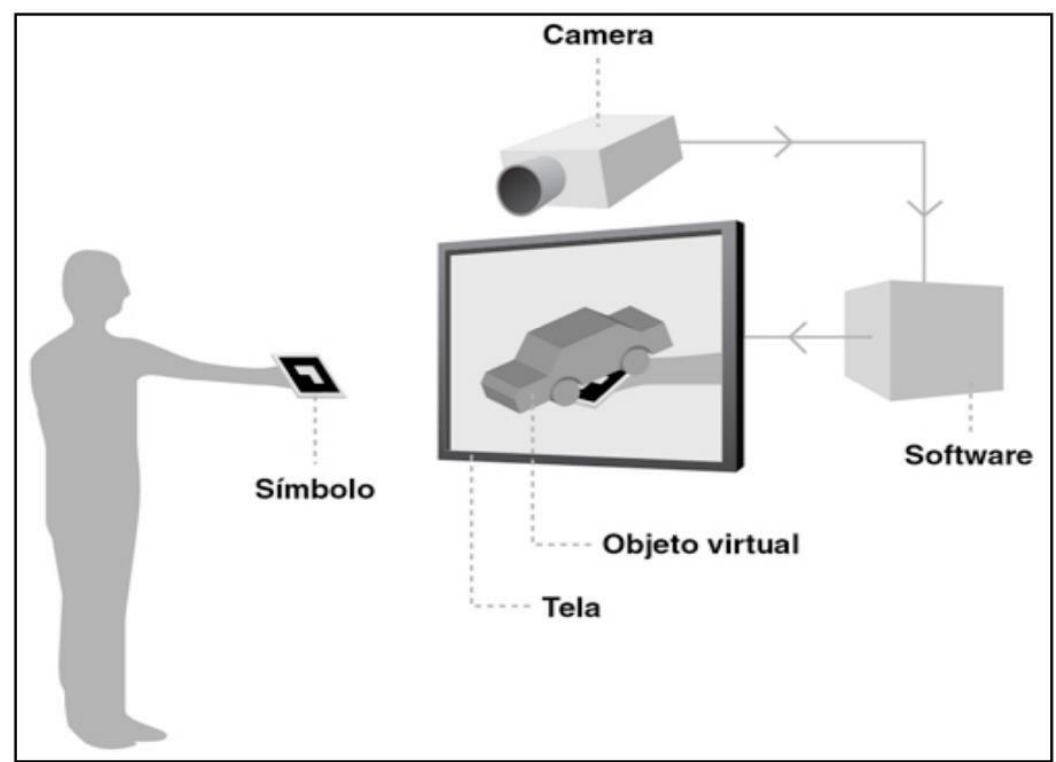

Figura 1. Exemplo do funcionamento da aplicação em Realidade Aumentada Fonte [Cardoso et al 2014]

De acordo com a Figura 1, o uso da RA é demonstrado por meio do reconhecimento de símbolos (marcadores), em que estes marcadores são apontados para a área de atuação da câmera, em seguida processada por um software que detecta esses símbolos onde os objetos virtuais são sobrepostos e por fim exibidos em algum dispositivo de saída, sejam eles televisão, telas de smartphones, ou datashows. A Figura 2 demonstra um marcador de identificação em Realidade Aumentada.

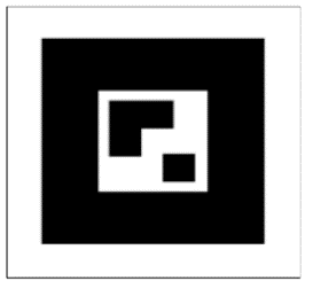

Figura 2. Exemplo de marcador de informação em Realidade Aumentada

\section{Fonte [Cardoso et al 2014]}

O marcador que é previamente agregado ao sistema RA, geralmente é criado em contrastes de preto e branco e com desenhos retangulares, pois é facilmente processado pelo software criado. A exibição do marcador fisicamente impresso em direção à câmera, é a conexão direta do meio real para a sobreposição dos objetos virtuais inseridos no software.

O Unity 3D é um ambiente de desenvolvimento integrado (IDE), voltado especificamente para o desenvolvimento de jogos, e aplicativos de visualização 3D, está em grande destaque como game engine (motor de jogo), pela sua fácil utilização, visualização do game em tempo de desenvolvimento e vários tutoriais na internet. As aplicações desenvolvidas nesta IDE podem ser disponibilizadas na web, em consoles para games, e em lojas de aplicativos móveis (Android e IOS), e computadores com sistemas operacionais Windows ou MAC OS. 
VI Congresso Brasileiro de Informática na Educação (CBIE 2017)

Anais do XXIII Workshop de Informática na Escola (WIE 2017)

A Vuforia é uma extensão da Unity3D em formato de Kit de Desenvolvimento de Software (SDK) que permite aos desenvolvedores criar facilmente aplicações RA. Com ela pode-se associar qualquer imagem ou código como um marcador de informação de RA de forma intuitiva. A Vuforia é uma ferramenta paga, mas não há custo inicial para o desenvolvimento ou para utilização com fins educacionais.

\section{Aplicativo Cubo Kids}

Com base nos conceitos apresentados na Seção 2 anterior, foi desenvolvido o aplicativo Cubo Kids, com o qual é possível suplementar o aprendizado infantil a nível de letramento, aprendizado das cores e animais, como também estimular as atividades lúdicas envolvendo os familiares na educação das crianças.

Foi elaborado através da IDE Unity3D, com a integração da Vuforia que por sua vez permitiu a inserção da RA aos dispositivos móveis. Aparelhos com câmera integrada, celulares ou tablets, com sistema operacional Android à partir da versão 2.3.1, API nível 9 são requisitos para utilização da ferramenta.

O aplicativo é gratuito e encontra-se disponível para baixar na loja virtual Google Play Store (https://play.google.com/store/apps/) buscando por 'CuboOut.CuboKids'. Os marcadores de identificação para interação com a realidade aumentada encontram-se na descrição do aplicativo. Exemplos deles são mostrados na Figura 3.

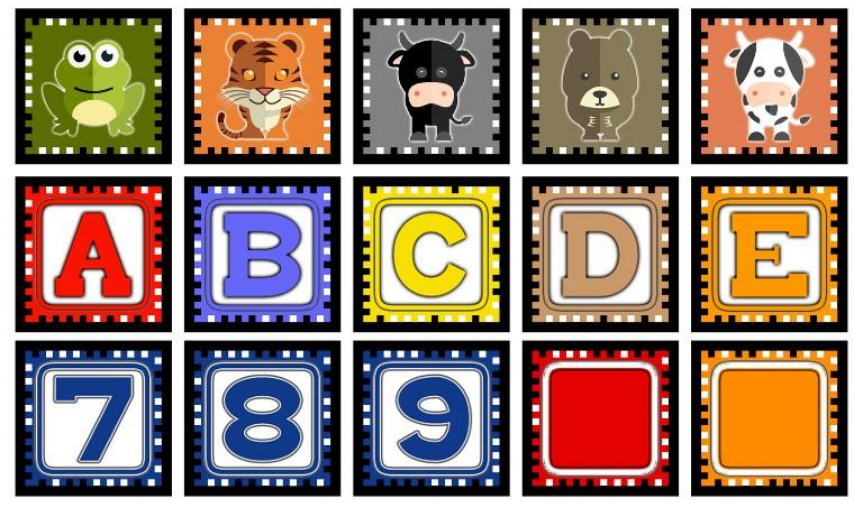

Figura 3. Marcador de letras em Realidade Aumentada Fonte Autoria própria

Para usar o Cubo Kids é necessário ter o aplicativo instalado em um aparelho Android, e ter os marcadores únicos. A tela inicial do aplicativo Cubo Kids, apresentada na Figura 4, é simples e intuitiva, composta por um único botão escrito "APRENDER BRINCANDO" onde é possível inicia-lo apenas tocando sobre o botão. 
VI Congresso Brasileiro de Informática na Educação (CBIE 2017)

Anais do XXIII Workshop de Informática na Escola (WIE 2017)

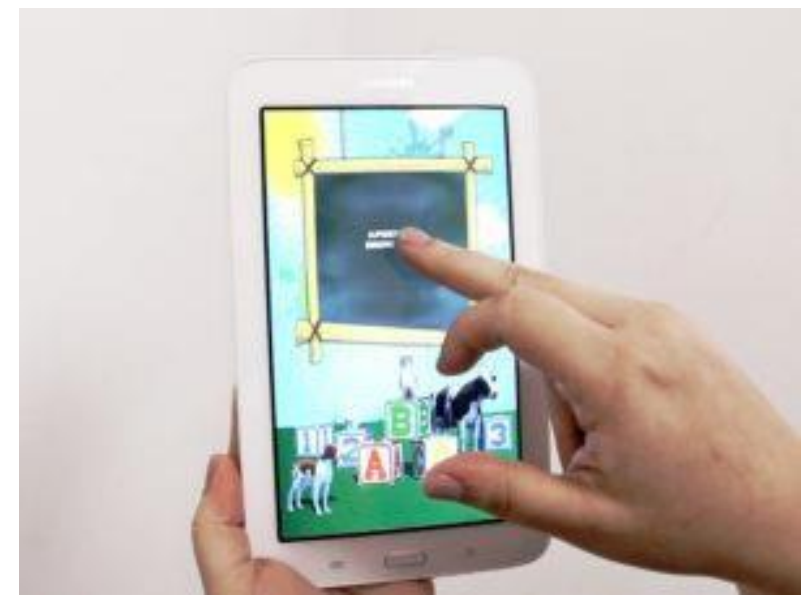

Figura 4. Tela inicial do aplicativo Fonte Autoria própria

A Figura 5 mostra os marcadores sem a sobreposição de objetos em RA à esquerda, e com realidade aumentada pelo aplicativo Cubo Kids à direita. Cada marcador de letra está sobreposto com um cubo e sua respectiva letra em três dimensões (3D). Para a projeção 3D acontecer é simples, basta apontar a câmera para o marcador, que o aplicativo vai identificar qual objeto 3D será sobreposto.

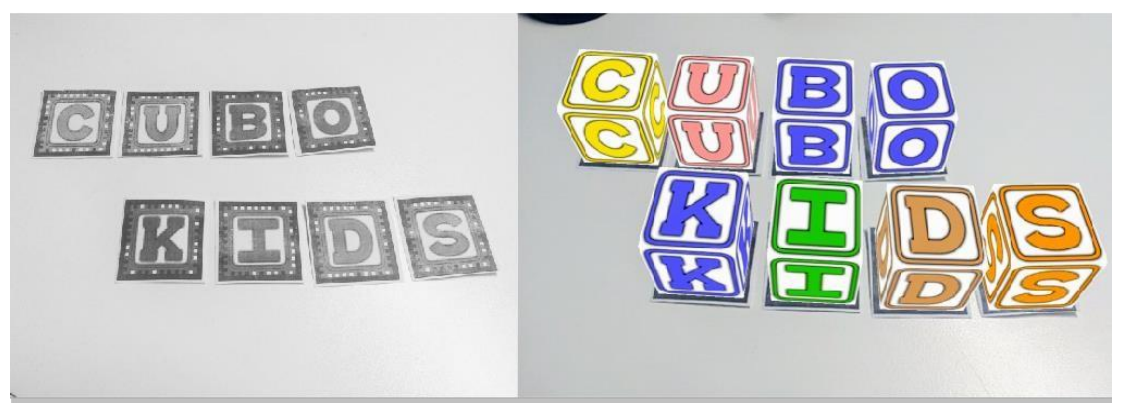

Figura 5. Marcador de letras em Realidade Aumentada

Cada objeto virtual depois de instanciado na tela do dispositivo tem uma interação ao tocar na tela sobre o objeto. Quando o objeto é letra, é mostrado algum objeto sobre o cubo, que inicia com a letra correspondente e é apresentado o som da letra. Quando se trabalha com a imagem de um animal, é emitido o som e apresentando o movimento do animal, além do nome do animal na língua inglesa. Ao se projetar um número, é apresentado como este é representado na Língua Brasileira de Sinais. Por fim, quando se apresenta uma cor, o nome da cor é emitido. Exemplos de interações são mostrados na Figura 6 com letra, número, cor e animal. 


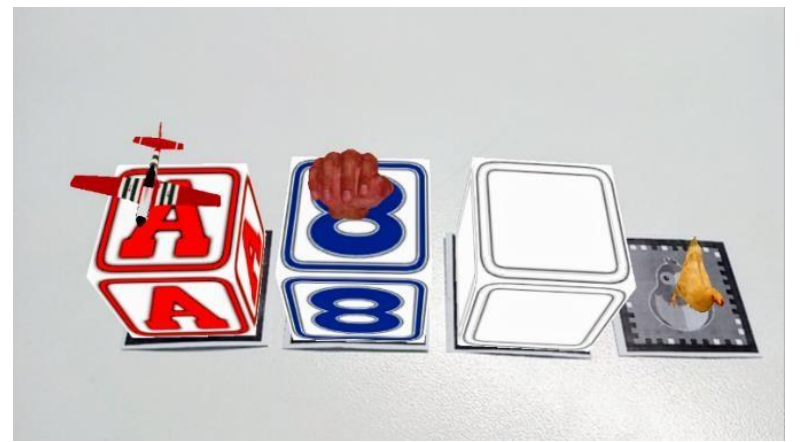

Figura 6. Objetos instanciados e com interação em Realidade Aumentada Fonte Autoria própria

O Cubo Kids está inserido no ensino de crianças ainda em fase de letramento/alfabetização, crianças estas que estão aprendendo e conhecendo os animais, as letras, os números, as cores e possuem idade entre dois e seis anos. A ferramenta é ideal para interação do público infantil com seus responsáveis educadores, sejam eles pais, professores ou responsáveis. A Seção 4 a seguir apresenta como esta foi utilizada como ferramenta de aprendizagem móvel com realidade aumentada para Educação Infantil.

\section{Estudo de Caso}

Para o desenvolvimento deste trabalho, foi realizada uma pesquisa qualitativa por ser uma metodologia que melhor representa os estudos nos aspectos humanos e sociais [Bogdan e Biklen, 1994]. Aliada a esta pesquisa, foi feita uma experiência in loco, com o uso da ferramenta Cubo Kids, em um ambiente real de aprendizagem, com o intuito de avaliar e validar a ferramenta, tendo como público-alvo alunos e professores da Educação Infantil e Ensino Fundamental.

\section{1- Contexto da pesquisa}

A pesquisa foi realizada em uma escola na cidade de Mossoró-RN. As experiências in loco foram realizadas em dez turmas da Educação Infantil (Infantil 1 ao Infantil 5), dos turnos matutino e vespertino, com um total de 20 professoras e cerca de 150 crianças participantes, com faixa etária entre dois e seis anos de idade.

Para o desenvolvimento da pesquisa e da experiência in loco, primeiramente foi realizada uma reunião com a equipe pedagógica da escola para apresentar o aplicativo Cubo Kids. Após isso, foi realizada uma demonstração de uso do aplicativo a todas as professoras, ensinando-as como utilizá-lo, de forma que cada uma pudesse, de acordo com o contexto de sala de aula, faixa etária de seus alunos e os conteúdos vistos em sala, desenvolver uma metodologia que pudesse aplicar a ferramenta durante a aula.

\section{2- Experiência in loco}

As experiências in loco ocorreram durante três semanas, de forma que todas as turmas descritas no tópico anterior participaram das ações. Para que cada ação ocorresse de forma efetiva, as professoras desenvolveram seu plano de aula, abordando assuntos 
pertinentes, de acordo com a série, faixa etária dos alunos, e os conteúdos que estavam sendo vistos em sala de aula e que pudessem ser complementados com o uso do aplicativo Cubo Kids.

Para a realização das experiências in loco, foram levados para as escolas smartphones e tablets com a ferramenta instalada para que os alunos pudessem fazer uso dos dispositivos móveis no contexto escolar para o seu aprendizado.

A Tabela 1 apresenta por série escolar a metodologia desenvolvida pelas professoras para o uso da ferramenta, quantas professoras e quantos alunos participaram da experiência.

Tabela 1: Séries, metodologias e participantes da experiência in loco

\begin{tabular}{|l|l|l|c|}
\hline \multicolumn{1}{|c|}{ Série } & \multicolumn{1}{|c|}{ Metodologia Usada } & \multicolumn{1}{|c|}{$\begin{array}{c}\text { Total } \\
\text { Professoras }\end{array}$} & $\begin{array}{c}\text { Total } \\
\text { alunos }\end{array}$ \\
\hline $\begin{array}{l}\text { Infantil 1 } \\
\text { A e B })\end{array}$ & $\begin{array}{l}\text { Explorando os animais, sons e gestos dos animais. } \\
\text { Compreender a importância dos animais para o meio } \\
\text { ambiente. }\end{array}$ & 6 & 32 \\
\hline $\begin{array}{l}\text { Infantil 2 } \\
\text { A e B })\end{array}$ & $\begin{array}{l}\text { Explorando os animais, sons e gestos dos animais. } \\
\text { Animais domésticos X selvagens. } \\
\text { Animais pequenos X grandes. } \\
\text { Reconhecendo as cores. }\end{array}$ & 6 & 28 \\
\hline $\begin{array}{l}\text { Infantil 3 } \\
\text { (A e B })\end{array}$ & $\begin{array}{l}\text { Reconhecendo letras do nome. } \\
\text { Associando letras a objetos. } \\
\text { Quantidades. }\end{array}$ & 5 & 35 \\
\hline $\begin{array}{l}\text { Infantil 4 } \\
\text { (A e B })\end{array}$ & $\begin{array}{l}\text { Reconhecendo as letras do Alfabeto. } \\
\text { Reconhecendo animais e suas letras iniciais. } \\
\text { Reconhecendo números e associando a quantidades. }\end{array}$ & 4 & 34 \\
\hline $\begin{array}{l}\text { Infantil 5 } \\
\text { (A e B) }\end{array}$ & $\begin{array}{l}\text { Formando sílabas. } \\
\text { Reconhecendo animais e suas sílabas iniciais. }\end{array}$ & 4 & 30 \\
\hline
\end{tabular}

Em cada turma, todos os alunos puderam utilizar os smartphones ou tablets, de acordo com a metodologia definida pela professora. A carga horária de utilização do aplicativo em cada turma foi de duas horas/aula. Todas as professoras priorizaram o uso da ferramenta de forma coletiva e colaborativa. Desta forma, para um uso mais efetivo, o smartphone ou tablet utilizado na experiência in loco foi conectado a uma televisão, com alto falantes, para que todas as crianças vivenciassem ao mesmo tempo a experiência do uso da M-Learning com Realidade Aumentada.

\section{5- Resultados}

Para a coleta de dados e obtenção dos resultados, foram feitas observações durante as experiências in loco e aplicação de questionário às professoras. A proposta da coleta de dados é saber a viabilidade de se utilizar o Cubo Kids como uma ferramenta $M$ Learning, observar os comportamentos das crianças e professoras durante o uso, obter sugestões de melhorias, e verificar os ambientes nos quais ela pode ser utilizada.

Cada questionário possuía 13 questões fechadas, as quais as professoras podiam dar as seguintes opiniões: 1- Discorda totalmente, 2- Discorda, 3- Sem opinião, 4Concorda, 5- Concorda totalmente, e duas questões abertas. As perguntas elaboradas, e 
VI Congresso Brasileiro de Informática na Educação (CBIE 2017)

Anais do XXIII Workshop de Informática na Escola (WIE 2017)

as médias obtidas, de acordo com a opinião das 24 professoras que responderam ao questionário são apresentadas na Tabela 2.

Tabela 2: Questionário aplicado e resultados obtidos

\begin{tabular}{|l|l|}
\hline Questão & Média obtida \\
\hline O aplicativo Cubo Kids é voltado para o público infantil? & 5 \\
\hline $\begin{array}{l}\text { O aplicativo Cubo Kids foi utilizado de forma eficiente em sua } \\
\text { turma? }\end{array}$ & 5 \\
\hline $\begin{array}{l}\text { Você indicaria o aplicativo para outros professores de séries } \\
\text { equivalentes? }\end{array}$ & 5 \\
\hline $\begin{array}{l}\text { O aplicativo pode ser adaptado para outros contextos da faixa-etária } \\
\text { de seus alunos? }\end{array}$ & 5 \\
\hline O aplicativo é de fácil manuseio? & 4 \\
\hline $\begin{array}{l}\text { O aplicativo tem uma boa interface e fácil interpretação pelas } \\
\text { crianças? }\end{array}$ & 4 \\
\hline O aplicativo é atrativo? & 5 \\
\hline As crianças mostraram interesse na aula ao usar o aplicativo? & 5 \\
\hline $\begin{array}{l}\text { O conteúdo do aplicativo está de acordo com a faixa etária da sua } \\
\text { turma? }\end{array}$ & 5 \\
\hline $\begin{array}{l}\text { Suas habilidades com o uso de tecnologias digitais possibilitam que } \\
\text { você use o aplicativo? }\end{array}$ & 4 \\
\hline As crianças se divertiram com o aplicativo? & 5 \\
\hline A experiência de utilizar o aplicativo em sala de aula foi positiva? & 5 \\
\hline O aplicativo contribuiu para a aprendizagem do seu aluno? & 5 \\
\hline
\end{tabular}

De acordo com os dados obtidos com a aplicação do questionário, todas as professoras concordam ou concordam totalmente com as perguntas feitas a respeito da utilização do Cubo Kids como aplicativo para M-Learning. Isso mostra que, de fato, o aplicativo apresentado neste artigo pode ser utilizado como mediador da aprendizagem nas séries iniciais, de forma a promover o processo de ensino e aprendizagem de maneira lúdica, em que a criança pode aprender brincando.

Além das questões fechadas, foram realizadas duas perguntas às professoras, as quais são apresentadas a seguir:

- Em que eixos temáticos educacionais o Cubo Kids se encaixa?

- Que sugestões você daria para que o aplicativo fosse melhorado?

Em relação à primeira pergunta as professoras pontuaram os seguintes eixos: natureza e cultura; linguagem oral; desenvolvimento lógico matemático; saberes científicos e tecnológicos; natureza e sociedade; artes; visão de mundo e meio ambiente. Como resultado a essa pergunta, vê-se que o aplicativo é adaptável à vários contextos e eixos educacionais, de forma que pode ser explorado em vários aspectos, de acordo com o planejamento e metodologia desenvolvida pelo professor.

Para a segunda pergunta, algumas sugestões foram fornecidas pelas professoras. A saber: ampliar as imagens; variar as imagens que representam as letras; trabalhar com formas geométricas; que os números apresentem suas quantidades correspondentes; trabalhar corpo humano; trabalhar alimentos. 
As sugestões dadas pelas professoras são de grande valia, e serão acatadas à medida do possível, para que outras áreas temáticas possam ser contempladas com o uso da ferramenta proposta.

Através da observação das experiências in loco, percebeu-se que tanto as crianças quanto as professoras sentiram-se confortáveis para utilizar a ferramenta. Todas as crianças demonstraram interesse, curiosidade, expectativa e atenção ao utilizar o aplicativo em sala de aula. As professoras relataram que, por ter a tecnologia de Realidade Aumentada, o Cubo Kids apresentava um diferencial e chamava mais atenção às crianças que, por meio dele, tinham melhor noção de movimento e sons dos objetos, além de reconhecimento das letras e associação de nomes, cores, animais, a elas.

\section{Considerações Finais}

Pode-se ver o quanto que a utilização de dispositivos móveis com finalidades educativas vem crescendo a cada dia. Aliada a ela, nota-se o quanto a RA pode contribuir no desenvolvimento de aplicativos na área da Educação. A partir de suas características de usabilidade, dinamismo, capacidade de promover a interação entre os mundos real e virtual, torna a capacidade de aprender e ensinar fascinante diante da disposição de se ter o conhecimento no cotidiano em qualquer lugar.

Com isso, este trabalho discutiu a utilização da tecnologia de Realidade Aumentada combinada a aprendizagem móvel, através do aplicativo Cubo Kids, para criação de novas metodologias de ensino para a Educação Infantil permitindo ampliar as possibilidades pedagógicas.

O Cubo Kids propõe ser um auxílio a professores no tocante ao letramento, a fim de estimular os alunos a aprenderem conteúdos de sala de aula de forma lúdica de divertida, com o auxílio da tecnologia RA móvel, o que pôde ser validado na experiência in loco.

Como proposta de melhorias futuras propõe-se adicionar novas funcionalidades na aplicação, tais como, objetos aleatórios para as letras, quantidades para os numerais, novos animais. Além disso, pretende-se separar o aplicativo Cubo Kids por módulos, de forma a aumentar as possibilidades de aprendizagem e métodos de ensino, tais como estudo de alimentos, partes do corpo humano, flora, dentre outros.

\section{Referências}

Attewell, J., Blenkinsopp, A., \& Black, P. (2005). Community pharmacists and continuing professional development - a qualitative study of perceptions and current involvement. The Pharmaceutical Journal, 274, 519-524.

Bogdan, R.C.; Biklen, S.K. (1994) Investigação qualitativa em educação: uma introdução à teoria e aos métodos. Tradução Maria João Alvarez. Porto: Porto.

Cardoso, R. G., Pereira, S. T., Cruz, J. H., \& Almeida, W. R. (2014). Uso da realidade aumentada em auxílio à Educação. Anais do Computer on the Beach, 330-339.

Kirner, C. Evolução da Realidade Virtual no Brasil. In: X Simposio em Realidade Virtual e Aumentada, João Pessoa, PB, SBC, p. 1-11. 2008. 
VI Congresso Brasileiro de Informática na Educação (CBIE 2017)

Anais do XXIII Workshop de Informática na Escola (WIE 2017)

Kirner, C.; Pinho, M.S. Uma Introdução à Realidade Virtual. Disponível em: http://grv.inf.pucrs.br/Pagina/TutRV/tutrv.htm. Acesso em 22 de Janeiro de 2006.

Kukulska-Hulme, A.; Traxler, J.. Mobile Learning: A Handbook for Educators and Trainers. London: Routledge. 2005.

Lima, Moixilene Sales Santos; Lima, Lavina Lúcia Vieira Lima; Lima Neto, Clodomir Silva; Castro Filho, José Aires de. Experiência de Aprendizagem Móvel: o uso do WhatsApp no Ensino Fundamental. Anais dos Workshops do V Congresso Brasileiro de Informática na Educação. V Congresso Brasileiro de Informática na Educação. Belo Horizonte- MG. 2016.

Oliveira, Rafael Costa de; Silva, Danila; Fernandes, Flávia Gonçalves; Oliveira, Luciene Chagas de; Oliveira, Eduardo Chagas de. Aplicativo de Aprendizagem Móvel utilizando Realidade Aumentada para Ensino de Língua Inglesa. Anais dos Workshops do V Congresso Brasileiro de Informática na Educação. V Congresso Brasileiro de Informática na Educação. Belo Horizonte - MG. 2016.

Saccol, A.; Shlemmer, E. ; Barbosa, J. (2011) M-learning e u-learning: Novas perspectiva das aprendizagens móvel e ubíqua. São Paulo: Pearson Prentice Hall.

Sutherland, Ivan. Sketchpad-A Man-Machine Graphical Communication System, Proceedings of the Spring Joint Computer Conference, Detroit, Michigan, May 1963 (Washington, D.C.: Spartan, 1964).

Tori, R.; Kirner, C.; Siscouto, R..Fundamentos e Tecnologia de Realidade Virtual e Aumentada. Belém: VIII Symposium On Virtual Reality, 2006.

Woodill, G. The Mobile Learning Edge: Tools and Technologies for Developing Your Teams, 1st ed., McGraw-Hill, Ed., 2010. [Online]. Disponível em: http://www.mobilelearningedge.com. 